\title{
Impacto de la Educación Emprendedora en Intención de Emprendimiento en Universidades del Noroeste de México.
}

\section{Impact of Entrepreneurial Education on Entrepreneurship Intention in Universities in the Northwest of Mexico.}

\author{
Clark Corral Jesús Hiram¹, Carrillo Peñuelas Dulce María ${ }^{2}$, Leyva Osuna Beatriz Alicia ${ }^{3}$ \\ ${ }^{1}$ Domicilio: Eucalipto $1117 \mathrm{col}$ chapultepec \\ Celular: 6441026603 \\ Jesus_clark_corral@hotmail.com \\ Egresado de Instituto Tecnológico de Sonora \\ ORCID: https://orcid.org/0000-0002-5345-9523
}

${ }^{2}$ Domicilio: Hilario Medina \#377 col. Sochiloa

Celular: 6442276379

Correo: dulce.carrillo185060@ potros.itson.edu.mx

Egresada Instituto Tecnologico de Sonora

ORCID ID: https://orcid.org/0000-0001-8206-7679

${ }^{3}$ Domicilio: 05 de febrero 818 sur, Col. Centro

Celular: 6441413448

beatriz.leyva@itson.edu.mx

Doctora en Filosofía con Especialidad en Administración por la Autónoma de Nuevo León, Maestra en Administración y Licenciada en Administración por el Instituto Tecnológico de Sonora, Profesor-Investigador de tiempo completo de Instituto Tecnológico de Sonora

ORCID: https://orcid.org/0000-0003-4935-6326

\section{DOI: https://doi.org/10.46589/rdiasf.vi36.409}

Recibido 14 de junio 2021.

Aceptado 29 de septiembre 2021

Publicado 1 de noviembre 2021 


\title{
Resumen
}

Se llevó a cabo un estudio cuantitativo que permite Analizar las relaciones entre las variables de actitud, normas subjetivas, control del comportamiento, educación empresarial, e intención empresarial de algunas universidades al Noroeste de México, con objetivo: Analizar a través de una investigación empírica el impacto que tiene la Educación Emprendedora en la intención de emprender de los alumnos de las Universidades del Noroeste de México, que permita determinar el efecto de los cursos de emprendimiento en los jóvenes. Se aplicó un instrumento de 27 preguntas con formato electrónico elaborado en google forms. El análisis estadístico se llevó a cabo en el software SMART-PLS (Ecuaciones Estructurales), obteniendo los siguientes resultados: Se comprueban seis de las siete hipótesis establecidas, rechazándose la relación de control comportamiento con la intención emprendedora, con los siguientes datos ( $\beta 0.090$ con un Pvalor 0.054), siendo la variable de actitud la más altamente significativa en su relación con la educación emprendedora ( $\beta 0.335$ con un Pvalor 0.000 ), y en segundo lugar de importancia la relación entre la Educación Emprendedora con la Intención Emprendedora. ( $\beta 0.333$ con un Pvalor 0.000). Se comprueba de manera empírica y teórica la mayoría de las hipótesis.

Palabras Claves: Comportamiento Planeado, Emprendedurismo, Iniciativa

\begin{abstract}
A quantitative study was carried out that allows to analyze the relationships between the attitude variables, subjective norms, behavior control, business education, and business intention of some universities in the Northwest of Mexico, with the objective: to analyze through empirical research the impact that Entrepreneurship Education has on the entrepreneurship intention of the students of the Universities of the Northwest of Mexico, which allows determining the effect of entrepreneurship courses on young people. An instrument of 27 questions with electronic format elaborated in google forms was applied. Statistical analysis was carried out in SMART-PLS software (Structural Equations), obtaining the following results: six of the seven established hypotheses are tested, rejecting the behavior control relationship with the entrepreneurial intention, with the following data ( $\beta 0.090$ with a P-value 0.054$)$, the attitude variable being the most highly significant in its relationship with entrepreneurial education ( $\beta 0.335$ with a Pvalue 0.000$)$, and secondly, the relationship with entrepreneurial education and entrepreneurial intention. ( $\beta$ 0.333 with a $\mathrm{P}$ value 0.000 ). Most hypotheses are tested empirically and theoretically.
\end{abstract}

\section{Keywords}

Planned Behavior, Entrepreneurship, Initiative

ISSN: 2007-8870

Universidad de Sonora
Los contenidos de este artículo están bajo una licencia de Creative Commons Atribución No Comercial - Sin Obra Deriva da 4.0 internacional 


\section{Introducción}

La teoría del comportamiento planificado ha retomado un gran auge, entre las investigaciones con relación al tema de emprendimiento, dicha teoría está conformada por las variables de actitudes, normas subjetivas y el control del comportamiento percibido (Ajzen, 1991), citado por Martínez, Salazar \& Mendoza (2020); por ello la importancia de la aplicación en un área de interés especificamente su aplicación en las universidades para obtener información necesaria para entender el comportamiento que presentan los alumnos e incluso implementar acciones que mejoren las condiciones y motivación de los futuros profesionistas.

Figura 1. Modelo de la Teoría de Comportamiento Planificado

Es el grado en el
que el individuo se
evalúa sobre ser
emprendedor
Es cómo el
individuo se
percibe ante la
facilidad o facultad
de convertirse en
emprendedor

Es cómo el
individuo percibe
que las personas
significativas
aprobarían la
decisión de
convertirse en
empresario
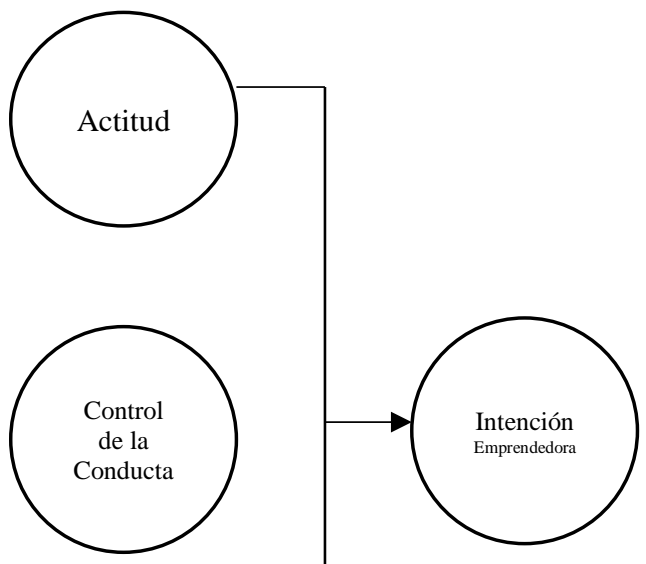

Fuente: Ajzen (1985) citado por Contreras \& Macías (2021). 
En México se analizaron diversos factores para determinar las condiciones de emprender por estado, al tener los resultados se clasificaron cuatro niveles de condiciones de emprendimiento. Solamente un estado entra en condiciones altas, siendo la Ciudad de México; con un nivel medio/alto entran el Estado de México, Jalisco y Veracruz; Chiapas, Guanajuato, Puebla y Yucatán se encuentran en condiciones medias; por último, con condiciones bajas están los 24 estados restantes, lo cual representa el $81 \%$ del país. Lo anterior da a entender que solo una pequeña parte del país cuenta con una mejor oportunidad de emprender, que tenga altas condiciones en su entorno no significa que automáticamente un negocio nuevo va a tener éxito, así como que el nivel sea bajo signifique que fracasará. (García, Zerón, y Sánchez, 2018).

Según Coto (2018) un $87 \%$ de los mexicanos no emprenden debido al miedo a fracasar, ya sea en un sentido económico o legal y si se llega a emprender negocios, el 75\% de estos cierran y aproximadamente el $10 \%$ tendrán más de 10 años de vida en el mercado. Una de las principales razones de que esto pasa esto es debido a la falta de programas educativos con un enfoque al emprendeurismo.

Otro problema es que las personas al tener la idea de emprender, no saben a dónde dirigirse, debido a lo mismo que no hay una cultura educativa de emprender y además no conocen programas de apoyo, los cuales impulsan a los mexicanos a empezar un negocio y hacerlo sobrevivir (Meza, 2016). En la tabla 1 se presentan las cifras de empresas supervivientes y su mortalidad.

Supervivientes y Mortalidad acumulada por cada 100 negocios que ingresan a la actividad económica por sector económico, según edad de las empresas. Datos a nivel Nacional.

\begin{tabular}{ccccc}
\hline $\begin{array}{c}\text { Edad de los } \\
\text { negocios }\end{array} \quad$ Mortandad & $\begin{array}{c}\text { Servicios } \\
\text { privados no } \\
\text { financieros }\end{array}$ & Manufacturero & Comercio \\
\hline
\end{tabular}




\begin{tabular}{ccccc}
\hline 0 & - & 100 & 100 & 100 \\
1 & 33 & 68 & 70 & 66 \\
5 & 65 & 36 & 40 & 33 \\
10 & 76 & 26 & 30 & 23 \\
15 & 82 & 20 & 24 & 16 \\
20 & 86 & 15 & 20 & 12 \\
25 & 89 & 12 & 17 & 9 \\
\hline
\end{tabular}

Fuente: INEGI (2014). Censos Económicos 1989, 1994, 1999, 2004, 2009 y 2014

En la tabla anterior, se puede decir que entre más años en operación tengan los negocios, son más propensos a cerrar permanentemente. Esto muestra que cada vez más las empresas tienen más obstáculos o no se actualizan, lo que tiene como consecuencia que no sobrevivan en el mercado. Incluso con nuevos negocios emergentes, las empresas siguen disminuyendo.

Para que un negocio tenga más probabilidad de tener éxito, este debe de tener origen en el esfuerzo, pasión, motivación personal y capacidad física e intelectual, pero la realidad de muchos jóvenes es que emergen como emprendedores queriendo sobrevivir, lo que causa una ganancia a corto plazo o que crece poco en la mayoría de los casos. (Canales, Román y Ovando, 2017).

Borrayo, Valdez y Delgado (2019) en su estudio señala que los jóvenes universitarios en caso de tener un trabajo, estarían dispuestos a renunciar a dicho empleo para empezar un negocio propio, a lo que el $49 \%$ respondieron afirmativo, mientras que $31.8 \%$ duda en aceptar el riesgo. Lo anterior da a entender que si los jóvenes ven una oportunidad de emprender la tomarán, lo cual demuestra una cultura emprendedora. De acuerdo a esta problemática se elabora la siguiente pregunta de investigación: ¿Cuál es el impacto que tiene la Educación Emprendedora con la Intención de Emprendimiento, en la formación del alumno universitario como emprendedor?

Por la importancia que justifica el estudio a través de los antecedentes y problema, se determina llevar a cabo el siguiente Objetivo:

ISSN: 2007-8870

Universidad de Sonora Commons Atribución No Comercial - Sin Obra Deriva da 4.0 Internacional 
Analizar a través de una investigación empírica el impacto que tiene la Educación Emprendedora en la intención de emprender de los alumnos de las Universidades del Noroeste de México, que permita determinar el efecto de los cursos de emprendimiento en los jóvenes.

\section{Hipótesis}

De acuerdo a la teoría se formulan las siguientes hipótesis para el presente estudio:

H1. La Actitud tiene una relación positiva y directa en la Educación Emprendedora de los universitarios.

H2. La Actitud tiene una relación positiva y directa en la Intención Emprendedora de los universitarios.

H3. La Norma Subjetiva tiene una relación positiva y directa en la Educación Emprendedora de los universitarios.

H4. La Norma Subjetiva tiene una relación positiva y directa en la Intención de emprender de los universitarios.

H5. El Control del Comportamiento tiene una relación positiva y directa en la Educación Emprendedora de los universitarios.

H6. El Control del Comportamiento tiene una relación positiva y directa en la Intención de emprender de los universitarios.

H7. La Educación Emprendedora tiene una relación positiva y directa en la Intención de emprender de los universitarios.

\section{Método}

La presente investigación se basa en el método científico aplicado con enfoque cuantitativo, según Creswell (2003), expone que la investigación cuantitativa se basa en investigaciones enfocadas a lo empírico o métodos científicos, lo que permite desarrollar el conocimiento y el pensamiento de causa-efecto. Así también esta investigación es descriptiva y correlacional, descriptiva ya que se compone de la descripción, registro y análisis e interpretación de la naturaleza del fenómeno, según Best, citado por Tamayo, (2004); y correlacional por que ayuda a determinar la relación de unos

ISSN: 2007-8870

Universidad de Sonora Commons Atribución No Comercial - Sin Obra Deriva da 4.0 internacional 
factores con otros, estableciendo las relaciones estadísticas de los fenómenos a estudiar. Tamayo (2004). El diseño de la Investigación es No Experimental y Transaccional, porque los sujetos o condiciones en la investigación no se manipulan, así también la investigación se realizará en un único momento, (Toro \& Parra, 2006).

La población a estudiar en el presente trabajo de investigación, son los alumnos universitarios del norte de México, que hayan cursado alguna materia que tenga que ver con emprendedurismo. Los alumnos deberán estar cursando una carrera en Administración o a fin. No hay muestra ya que se pretende entrevistar al total de los grupos de la cohorte del semestre de cada Universidad participante en este estudio, lo cual suma un total de 491 estudiantes.

El instrumento utilizado permite obtener datos con respecto al propósito de la investigación y a través del mismo se obtendrá la información para aceptar o rechazar las hipótesis planteadas. Se elabora el cuestionario de 23 preguntas que abarcan las variables de actitud, normas subjetivas, control de comportamiento, educación emprendedora e intención para emprender, de manera electrónica a través de google forms. El instrumento se adaptó de la investigación realizada por Fernández y Quintero (2017) lo que permite determinar que el instrumento está validado.

\section{Procedimiento}

Buscar información bibliográfica y en base de datos científicas que permitan consultar artículos referentes a las variables de investigación.

Llevar acabo el trabajo de campo, en las universidades participantes en el estudio, para crear una base de datos en el Sistema Estadístico SPSS versión 21, que permita obtener resultados de la aplicación del instrumento.

Para analizar los resultados y comprobar la hipótesis estadísticamente de la investigación, se utilizará el método de Ecuaciones Estructurales, ya que la bonanza de utilizarlo es que no exige la normalidad en los datos recolectados, Chin (1998) citado por Rositas (2005), como lo requiere el de Regresión. Para desarrollar esta modelación se utilizará el software SMART-PLS (Ecuaciones Estructurales).

ISSN: 2007-8870

Universidad de Sonora 
Elaborar conclusiones y recomendaciones referentes al análisis e interpretación de la información.

\section{Resultados}

Se presentan a continuación los resultados de la investigación (Análisis Demográfico del perfil del encuestado, Estadística Descriptiva, Análisis Estadístico), de la presente investigación aplicada a una muestra de 491 estudiantes al Norte de México.

El análisis estadístico se realiza a través de Ecuaciones Estructurales para facilitar la flexibilidad de los modelos de regresión, éstos incluyen errores de medida de las variables criterio (dependientes) y las predictoras (independientes), es así que puede pensarse en varios modelos para el análisis factorial, con efectos directos e indirectos en los factores (Ruiz, Pardo, \& San Martín, 2010). El procedimiento del SmartPLS es uno de los sistemas hasta el momento más utilizado para realizar modelos de ecuaciones estructurales. (Ringle, Wende, \& Will, 2005) citado por Kay (2013).



Modelo de Ecuaciones Estructurales. (Calculo Algoritmo)

Fuente: Adaptado con datos obtenidos del sistema estadístico SMART-PLS. (Ecuaciones Estructurales).

ISSN: 2007-8870

Universidad de Sonora 


\section{Primer Etapa: Validez y Confiabilidad}

\section{Resultados del análisis de Fiabilidad Compuesta}

\begin{tabular}{lll} 
& $\begin{array}{l}\text { Fiabilidad } \\
\text { compuesta }\end{array}$ & $\begin{array}{l}\text { Varianza } \\
\text { extraída media } \\
\text { (AVE) }\end{array}$ \\
\hline Actitud & 0.945 & 0.810 \\
$\begin{array}{l}\text { Norma Subjetiva } \\
\text { Control del }\end{array}$ & 0.892 & 0.734 \\
$\begin{array}{l}\text { Comportamiento } \\
\text { Educación }\end{array}$ & 0.754 & 0.606 \\
$\begin{array}{l}\text { Emprendedora } \\
\begin{array}{l}\text { Intención } \\
\text { Emprendedora }\end{array}\end{array}$ & 0.937 & 0.600 \\
\hline
\end{tabular}

Fuente: Elaboración propia, adaptado de los datos obtenidos del sistema estadístico SMART-PLS. (Ecuaciones Estructurales).

La confiabilidad compuesta mide los indicadores que conforman una variable latente a través de su consistencia interna, ésta se acepta en investigaciones de tipo exploratoria y sus rangos de valor para que sea aceptable deben ser por arriba de .60, (Nunnally y Bernstein, 1994). En cuanto a la varianza extraída (AVE), para explicar el grado de validez de un constructo debe ser arriba de .50, según la tabla arriba las variables latentes explican más de la mitad de la varianza de los indicadores que la componen. (Hair, Ringle y Sarstedt, 2011). 


\section{Resultados del análisis de medición: cargas}

\begin{tabular}{|c|c|c|c|c|c|}
\hline & Actitud & $\begin{array}{l}\text { Norma } \\
\text { Subjetiva }\end{array}$ & $\begin{array}{l}\text { Control del } \\
\text { Comportamiento }\end{array}$ & $\begin{array}{l}\text { Educación } \\
\text { Emprendedora }\end{array}$ & $\begin{array}{l}\text { Intención } \\
\text { Emprendedora }\end{array}$ \\
\hline A1 & .833 & & & & \\
\hline $\mathrm{A} 2$ & .917 & & & & \\
\hline A3 & .925 & & & & \\
\hline A4 & .922 & & & & \\
\hline N5 & & .875 & & & \\
\hline N6 & & .861 & & & \\
\hline N7 & & .834 & & & \\
\hline $\mathrm{C} 14$ & & & .766 & & \\
\hline $\mathrm{C} 15$ & & & .790 & & \\
\hline E23 & & & & .724 & \\
\hline E24 & & & & .771 & \\
\hline E25 & & & & .758 & \\
\hline E26 & & & & .795 & \\
\hline E27 & & & & .755 & \\
\hline E29 & & & & .745 & \\
\hline E30 & & & & .797 & \\
\hline E31 & & & & .816 & \\
\hline E32 & & & & .779 & \\
\hline E33 & & & & .803 & \\
\hline IN34 & & & & & 1.000 \\
\hline
\end{tabular}

Fuente: Elaboración propia, adaptada de los datos obtenidos del sistema estadístico SMART-PLS (Ecuaciones Estructurales).

Según Chin (1998) se aceptan las cargas arriba de .70. Se visualiza las cargas de acuerdo a los 20 ítems del modelo en los siguientes porcentajes divididos de la siguiente manera: de .700 se representa con el (50\%) de los Ítems, con cargas de .800 es el (30\%), con cargas arriba de .900 es un $(15 \%)$ y por último con 1.000 solo es el (5\%). Las cargas están en su mayoría en los niveles de .700 y .800 . 


\section{Resultados Coeficientes Path}

\begin{tabular}{lll} 
& $\begin{array}{l}\text { Educación } \\
\text { Emprendedora }\end{array}$ & $\begin{array}{l}\text { Intención } \\
\text { Emprendedora }\end{array}$ \\
\hline Actitud & .335 & .127 \\
Norma Subjetiva & .166 & .115 \\
& & \\
$\begin{array}{l}\text { Control del } \\
\text { Comportamiento }\end{array}$ & .249 & .090 \\
$\begin{array}{l}\text { Educación } \\
\text { Emprendedora }\end{array}$ & & .333 \\
\hline
\end{tabular}

Fuente: Elaboración propia, adaptada de los datos obtenidos del sistema estadístico SMART-PLS (Ecuaciones Estructurales).

Es importante mencionar que los valores de betas expuestos por Rositas (2005), van como se expone, para un valor de 0.00 a $0.09=$ imperceptible, 0.10 a $0.15=$ perceptible, 0.16 a $0.19=$ considerable, 0.20 a $0.29=$ importante, 0.30 a $0.50=$ fuerte y Mayores a $0.50=$ muy fuerte. De acuerdo a estos rangos se puede determinar los resultados de los coeficientes.

\section{Efectos Indirectos}

\begin{tabular}{|c|c|c|c|c|c|}
\hline & Actitud & $\begin{array}{l}\text { Normas } \\
\text { Subjetivas }\end{array}$ & $\begin{array}{l}\text { Control del } \\
\text { Comportamiento }\end{array}$ & $\begin{array}{l}\text { Educación } \\
\text { Emprendedora }\end{array}$ & $\begin{array}{l}\text { Intención } \\
\text { Emprendedora }\end{array}$ \\
\hline Actitud & & & & & 0.111 \\
\hline $\begin{array}{l}\text { Normas } \\
\text { Subjetivas }\end{array}$ & & & & & 0.055 \\
\hline $\begin{array}{l}\text { Control del } \\
\text { Comportamiento }\end{array}$ & & & & & 0.083 \\
\hline
\end{tabular}




\section{Segunda Etapa: Evaluación del Modelo Estructural}

Resultados de la $R^{2}$.

\begin{tabular}{lr} 
Variables & $\mathrm{R}^{2}$ \\
\hline Educación & \\
Emprendedora & 0.303 \\
Intención & \\
Emprendedora & 0.258 \\
\hline
\end{tabular}

Fuente: Adaptado de los datos obtenidos del sistema estadístico SMART-PLS. (Ecuaciones Estructurales).

Resultados de la $f^{2}$.

Actitud Normas Control del Educación Intención

Actitud Subjetivas Comportamiento Emprendedora Emprendedora 0.138 0.016

Normas 0.033 0.014

Subjetivas

Control del

0.080

0.009

Comportamiento

Educación

0.104

Emprendedora

Fuente: Adaptado de los datos obtenidos del sistema estadístico SMART-PLS. (Ecuaciones Estructurales).

Para Cohen (1998), indica el siguiente rango de valores para la f2 0.02 tiene efecto pequeño, 0.15 su efecto es mediano y 0.35 el efecto es considerable (grande), la f2 nos ayuda a evaluar si algún constructo omitido tiene un impacto considerable en constructos endógenos.

Los resultados de la $\mathrm{R}^{2}$ exponen que la variable de Educación Emprendedora se explica en un $30 \%$ por las variables latentes de Actitud, Normas Subjetivas y Control del Comportamiento, así también la variable de Intención Emprendedora se explica con un 25\%, por las variables de Educación Emprendedora, Actitud, Normas Subjetivas y Control del Comportamiento. 
Factor de Inflación de la varianza final (FIV)

\begin{tabular}{|c|c|c|c|c|c|}
\hline Variables & Actitud & $\begin{array}{l}\text { Norma } \\
\text { Subjetiva }\end{array}$ & $\begin{array}{l}\text { Control del } \\
\text { Comportamiento }\end{array}$ & $\begin{array}{l}\text { Educación } \\
\text { Emprendedora }\end{array}$ & $\begin{array}{l}\text { Intención } \\
\text { Emprendedora }\end{array}$ \\
\hline Actitud & & & & 1.161 & 1.322 \\
\hline $\begin{array}{l}\text { Norma Subjetiva } \\
\text { Control del } \\
\text { Comportamiento }\end{array}$ & & & & $\begin{array}{l}1.210 \\
1.117\end{array}$ & $\begin{array}{l}1.250 \\
1.206\end{array}$ \\
\hline $\begin{array}{l}\text { Educación } \\
\text { Emprendedora } \\
\text { Intención } \\
\text { Emprendedora }\end{array}$ & & & & & 1.434 \\
\hline
\end{tabular}

La Multicolinealidad permite determinar si existe redundancia en el modelo propuesto tomando como referencia sus indicadores, el Factor de Inflación de la Varianza (FIV) debe ser menor que 5, (Kutner, Nachtsheim \& Neter, 2004). De acuerdo a los resultados obtenidos se puede verificar que no hay multicolinealidad.

A través del cálculo de bootstrapping en sistema estadístico SMART-PLS, se lleva a cabo la prueba de hipótesis, a través de la t-Student y $\mathrm{P}$ (valor).

\section{Resultados del Análisis Estructural}

Muestra Estadístico P Valores

original $(\mathrm{O})$

.335

0.127

0.166

Control del Comportamiento-> Educación

Emprendedora

Control del Comportamiento-> Intención

Emprendedora

Educación Emprendedora-> Intención Emprendedora
0.115

0.249

0.090

0.333
3.368

2.126

5.368

\begin{tabular}{|c|c|c|}
\hline .223 & 0.000 & $\begin{array}{l}\text { Se Acepta } \\
* * *\end{array}$ \\
\hline 146 & 0.032 & $\begin{array}{l}\text { Se Acepta } \\
*\end{array}$ \\
\hline
\end{tabular}

0.001

Se Acepta $* * *$

$0.034 \quad$ Se Acepta

*

Se Acepta

$* * *$

$1.930 \quad 0.054 \quad$ No se Acepta

$\begin{array}{ll}0.000 & \begin{array}{l}\text { Se Acepta } \\ * * *\end{array} \\ 0.054 & \text { No se Acepta }\end{array}$

5.081

0.000

Se Acepta

$* * *$

Fuente: Adaptado de los datos obtenidos del sistema estadístico SMART-PLS. (Ecuaciones Estructurales).

ISSN: 2007-8870

Universidad de Sonora
Los contenidos de este artículo están bajo una licencia de Creative Commons Átribudión No Comercial - Sin Obra Deriva da 4.0 Internacional 
$\mathrm{P}$ value $* \mathrm{p}<=.05$, Significativo $* * \mathrm{p}<=.01$, Considerablemente significativo $* * * \mathrm{p}<=.001$ Altamente significativo, (Rositas, 2014).

Para la significancia en ecuaciones estructurales en SMART-PLS junto con los coeficientes (path), y la P valor se evalúa también la "t" student (p-valor) la cual mide la significancia de las relaciones donde si éstas llegan a 2.0 o lo superan es estadísticamente significativo (Hair, Ringle y Sarstedt, 2011). Lo que permite comprobar las hipótesis del estudio.

\section{Análisis y Discusión de Resultados en las Hipótesis}

La presente investigación aplicada a 491 estudiantes al Norte de México los cuales cursaron una materia de emprendimiento da como resultado la siguiente información con respecto a las hipótesis de la variable Actitud:

H1. La Actitud tiene una relación positiva y directa en la Educación Emprendedora de los universitarios.

H2. La Actitud tiene una relación positiva y directa en la Intención Emprendedora de los universitarios.

La hipótesis de este estudio fue aceptada con una significancia importante de $\beta$ (.335) y p- valor (.000) considerablemente significativos, con una relación positiva donde se establece que a mayor actitud mayor es mayor el impacto en la educación emprendedora en los alumnos universitarios. Lo que se refiere a la actitud y su relación con la intención emprendedora se observa que se tiene una relación positiva y directa, pero con una $\beta$ (.127) perceptible, y su p-valor (0.032) significativo.

También se observa que la actitud es el primer constructo con un papel importante en el modelo, sin embargo, la $f^{2}(0.138)$, indica que su participación (efecto) en el modelo es importante su influencia con la educación emprendedora, a su vez la varianza se explica con un $81 \%$ quedando respaldado que los indicadores explican fuertemente a su constructo. Si embrago la actitud tiene un efecto muy pequeño $f^{2}(0.016)$, en relación con la intención emprendedora. Los alumnos muestran una predisposición favorable hacia la materia de Emprendimiento. Cabe señalar que el efecto indirecto de la Actitud hacia la Intención Emprendedora no es muy fuerte (.111). 
A continuación, se presentan algunos estudios empíricos con relación a la hipótesis estudiada.

Los investigadores Azanza y Campos (2014) encontraron de manera positiva y significativa una relación entre actitudes emprendedoras y la intención de emprender con $(r=, 42, p<, 01)$, las universidades trabajan para crear una actitud favorable hacia el emprendimiento, la cual fortalecen a través de la educación emprendedora, con la finalidad de aumentar el número de alumnos que puedan identificar al autoempleo como una alternativa racional y viable donde puedan desarrollar su profesión. Alanzya (2012) expone que las actitudes enfocadas al emprendimiento desde jóvenes, tienen potencial en las habilidades que para cuando son adultos el emprendimiento tiene mayor éxito, por ello se debe fomentar programas en las escuelas de educación básica y media, con la finalidad de desarrollar futuros emprendedores. Sin embargo, en las universidades existe la necesidad de empatar la información teórica con la práctica, ya que un estudio realizado por Kirby (2002), citado por Sánchez, Ward, Hernádez y Florez (2017) en su estado del arte de la Educación emprendedora, expone que la educación emprendedora puede causar un efecto negativo en el desarrollo de las competencias y actitudes hacia el emprendimiento.

H3. La Norma Subjetiva tiene una relación positiva y directa en la Educación Emprendedora de los universitarios.

H4. La Norma Subjetiva tiene una relación positiva y directa en la Intención de Emprender de los universitarios.

Las hipótesis referentes a las normas subjetivas obtienen el siguiente resultado: la $\mathrm{H} 3$ fue aceptada con una relación positiva y directa, su $\beta$ (.166) y p-valor (.001) considerablemente significativos, en su impacto en la variable de educación emprendedora de los alumnos universitarios. Lo que se refiere a la $\mathrm{H} 4$ se determinó que existe una relación positiva y directa, con una $\beta$ (.115) perceptible, y su p-valor (0.034) significativo. Las normas subjetivas influyen por el apoyo de quedan los padres, amigos y personas importantes en las vidas de los alumnos universitarios que los motiva en su educación de emprender, así como en la intención de emprendimiento. 
El efecto que causan las Normas Subjetivas, es de $f^{2}(0.033)$, indica que su participación en el modelo es baja para la educación emprendedora, y para la intención de emprender tiene un efecto de $f^{2}(0.014)$, demasiado pequeño que apenas es perceptible en el modelo. Sin embargo, los indicadores que conforman su constructo lo explican en un $73 \%$ de su varianza. Cabe señalar que el efecto indirecto de las Normas Subjetivas hacia la Intención Emprendedora es demasiado bajo $(.055)$.

Algunos estudios empíricos con relación a la hipótesis estudiada con respecto a la variable Normas Subjetivas se presentan a continuación: según Contreras \& Macías (2021), en su estudio percepción de los estudiantes sobre la intención de emprender. Caso: Universidad de Guadalajara, México. Concluyen que las normas subjetivas aunque obtuvo un efecto positivo en el estudio no es significativa por lo que se rechaza la hipótesis, sin embargo la opinión de las personas más cercanas no impacta en la intención de emprender. En la Tesis "La relación de la educación empresarial con la intención emprendedora en las instituciones de educación superior del centro del país" en Perú; se obtiene como resultado que las normas subjetivas en los universitarios consideran que están más cercas de ellos y que son importanes no influyen en la decisión para ser emprendedor. (Salguero, 2018).

Para Osorio \& Londoño (2015) establecen que las normas subjetivas raramente predice las intenciones de emprender y en ocasiones los eliminan de los análisis de algunas investigaciones, esto sucede cuando los individuos poseen una alta capacidad de control interno y no necesariamente ocupan de las personas que están a su alrededor, según Ajzen (1987) citado por los ya mencionados autores. Los investigadores Bolívar, Lemes, y Medina, (2014), aclaran que existen investigaciones en las que se determina que las normas subjetivas están relacionadas fuertemente con los emprendedores no universitarios, según estos autores exponen que una explicación puede ser la situación económica que los lleva a tomar decisiones desesperadas por contar con un trabajo.

ISSN: 2007-8870

Universidad de Sonora 
H5. El Control del Comportamiento tiene una relación positiva y directa en la Educación Emprendedora de los universitarios.

H6. El Control del Comportamiento tiene una relación positiva y directa en la Intención de Emprender de los universitarios.

Las hipótesis referentes al control del comportamiento obtienen el siguiente resultado con respecto a la educación emprendedora, ésta fue aceptada con una relación (+) y directa, su $\beta(.249)$ y p-valor (.000) considerados fuertemente significativos. En lo referente al control de comportamiento con respecto a la intención de emprender existe una relación positiva y directa, con una $\beta$ (.090) imperceptible, y su p-valor (0.054) No significativo, por lo que esta hipótesis se rechaza. El efecto indirecto del control del comportamiento hacia la Intención Emprendedora es demasiado bajo (.083), por lo que no se considera una relación de impacto.

Con referencia al efecto que causan el control del comportamiento en el que los universitarios, donde creen en sus capacidades y en los recursos con los que cuentan es de $f^{2}(0.080)$, donde se indica que la participación en el modelo es baja para la educación emprendedora, y el efecto para la intención de emprender tiene un efecto de $f^{2}(0.009)$, casi nulo en el modelo. La varianza en el constructo se explica con un $60 \%$.

Torres y Vidal (2019), En su estudio llegan a la conclusión que las instituciones educativas a nivel superior deben determinar los valores que hacen que una persona sea decisiva, ya que éstos forman al individuo en su comportamiento y su conducta, y esto hace que ejecuten sus planes de manera más fácil; por ello las universidades deben enfocar sus materias en ofrecer a los universitarios una formación empresarial más efectiva y práctica, los ajustes en la materia deben ir enfocados a factores psicológicos, inteligencia emocional, entorno universitario, autoeficacia emprendedora, y por supuesto en experiencia laboral. Bolívar, Lemes, y Medina, (2014), encontraron que existe una relación (+) entre el control percibido del comportamiento y la variable de intención emprendedora, donde su relación se basa en que mayor autorreconocimiento de sus propias capacidades, incremente también la iniciativa por llevar a cabo aquello con lo que ellos sientan que su capacidad les permitirá ser competentes. 
El estudio de Morante (2018) muestra resultados que dejan claro que cuando un individuo no tiene iniciativa, o bien no buscan ser proactivos de manera inminente van directos a fracasar, dejando de lado las oportunidades y por lo tanto no perseveran, porque obviamente alguien con esas características no tendría la opción de emprender. Para los investigadores García, Mendoza, Romo, (2017) en su estudio la variable de control del comportamiento percibido, fue la más baja en comparación con la actitud y las normas subjetivas en relación con la intención de emprendimiento, lo que limita el desarrollo del alumno al comportamiento para ser emprendedor.

H7. La Educación Emprendedora tiene una relación positiva y directa en la Intención de Emprender de los universitarios.

La Educación Emprendedora, mantiene una relación (+) y directa, con una $\beta(0.333)$ y pvalor (0.000) considerados fuertemente significativos, con la intención de emprender.

De acuerdo a Sánchez et al. (2017) la importancia de la creación de programas enfocados a El peso de la labor de crear programas de educación emprendedora no solo es su diseño, lo importante es mantenerlos a través de una constante evalución que permita medir su viabilidad y efectividad que aumenten las competencias y su valor para la intención emprendedora de los alumnos. Se ha convertido en éxito la implementación del tema de emprendedores dentro de la currícula académica en las universidades, dichos programas deben estar adaptados a las demandas sociales, así mismo debens er tan transformacionales dentro de la pedagogía como para el negocio. (Bikse, Lusena, Rivza, \& Vilkova, 2016).

El 56\% de los universitarios de acuerdo a los resultados de la investigación Ramos, López, Moreno y Galván, (2018) expone que prefieren trabajar como empleados, y el resto que tiene la intención de contar con su propio negocio, las universidades deberían identificarlos y dar seguimiento para no desistan de su carrera empresarial, ya que definitivamente las universidades fomentan la intención de emprender, por lo que hay que orientar y seguir motivando para que no decaiga esa intención durante su estancia en la universidad.

ISSN: 2007-8870

Universidad de Sonora 


\section{Conclusión}

Se determina a través de este estudio que se alcanza el objetivo de la investigación, en la cual se concluyen lo siguiente:

En definitiva la Universidad tiene un compromiso fuerte ante la sociedad y su comunidad, esta tiene que ver guiar a sus alumnos para realizar su profesión y ésta le permita mantener calidad de vida a través del estudio. Dentro de estos compromisos y desarrollo de competencias se va integrando la posibilidad que los egresados no solo sean empleados, si no que tengan la posibilidad de iniciar su propio negocio el cual permita subsistir económicamente, es por ello que a través de éstas se promueve el espíritu emprendedor.

Para asegurar el éxito de éstos planes los mestros juegan papel fundamental junto con los programas de emprendimiento, los cuales deben cubrir el perfil para que se abarque lo teórico y lo empirico, así la motivación para el alumno será permanente durante toda su carrera con el deseo de el emprendimiento.

Algunas de las estrategias que pueden apoyar al éxito de mantener la intención de emprender es a través de la teoría de comportamiento planificado la cual abarca las variables de actitud, normas subjetivas y control del comportamiento que se analizan en este estudio, estas variables se pueden acompañar a través de talleres, conferencias, casos de éxito, instituciones gubernamentales y no gubernamentales involucradas para la creación de un negocio, y que permitan el desarrollo de estas variables en el papel de emprendedor.

Para el aseguramiento de la intención de emprender a través de la educación emprendedora, es interesante que se siguiera investigando en este tema para actualizar los programas universitarios investigando a los alumnos, a los empresarios y expertos en referencia a tema de comportamiento planificado, cultura, género, liderazgo, etc, haciendo los cruces de investigación.

Los resultados obtenidos en este estudio permiten evidenciar y validar la importancia de la variable de educación de emprendimiento con la teoría del comportamiento planificado que permita determinar una situación en universiades al norte de México.

Esta Investigación se deriva de un proyecto apoyado por el Programa para el Desarrollo Profesional Docente (PRODEP).

ISSN: 2007-8870

Universidad de Sonora Commons Atribución No Comercial - Sin Obra Deriva da 4.0 internacional 


\section{Referencias}

Alanzya, S. (2012). Habilidades sociales y actitud emprendedora en estudiantes del quinto de secundaria de una institución educativa del Distrito del Callao. Facultad de Educación, Programa de Maestría para docentes de la Región Calla. Recuperado de: http://repositorio.usil.edu.pe/bitstream/123456789/1080/1/2012_Alanya_Habilidadessociales-y-actitud-emprendedora-en-estudiantes-del-quinto-de-secundaria-de-unainstitucion-educativa-del-distrito-del-Callao.pdf

Azanza, G. y Campos, J. (2014). La intención emprendedora en estudiantes universitarios: el caso de la universidad de DEUSTO. Boletín de Estudios Económicos. 69(211). Pp 151175. Recuperado de: https://www.bizkailab.deusto.es/wpcontent/uploads/2014/06/Campos-AZANZA.pdf

Bikse, v., Lusena-Ezera, I., Rivza, B., Vo1kova, T. (2016). The Transformation of Traditional Universities into Entrepreneurial Universities to Ensure Sustainable Higher Education. Journal of Teacher Education for Sustainability, 18(2), 7588. https://doi.org/10.1515/jtes-2016-0016

Borrayo, C., Valdez, A. y Delgado, B. (2019). Cultura emprendedora en jóvenes universitarios de Guadalajara, México. https://dialnet.unirioja.es/servlet/articulo?codigo $=7026735$

Bolívar, A.; Lemes, A.; Medina, P. (2014). Un paso más en la intención emprendedora del estudiante universitario: GUESS. Revista Estudios Empresariales. Segunda Edición. (2). Pp:63-80. Recuperado de: file:///C:/Users/beatriz.leyva/Documents/2020/TESIS\%202020/Calculos/articulos/Dialnet -UnPasoMasEnLaInvestigacionDeLaIntencionEmprendedor-5098411.pdf

Canales, R., Román, Y. y Ovando, W. (2017). Emprendimiento de la población joven en México. Una perspectiva crítica. Recuperado de: http://revistas.unam.mx/index.php/entreciencias/article/view/62142/54696

Chin, W. (1998). The partial least squares approach to structural equation modeling. Modern methods for business research. 295-335.

Cohen, J. (1998). Statically power analysis for the behavioral sciences. Estados Unidos, New York: Laurence Erlbaum Associates. 
Contreras Cueva, A., \& Macías Álvarez, P. (2021). Percepción de los estudiantes sobre la intención de emprender. Caso: Universidad de Guadalajara, México. SUMMA. Revista Disciplinaria En Ciencias económicas Y Sociales, 3(1), 1-25. Recuperado de: https://doi.org/10.47666/summa.3.1.03

Coto, D. (2018). Resulta que México es el paraíso para emprender pero no para los millennials. Recuperado de: HTTPS://NEGOCIOSINTELIGENTES.MX/RESULTAQUE-MEXICO-ES-EL-PARAISO-PARAEMPRENDER-PERO-NO-PARA-LOSMILLENNIALS/

Creswell, John. (2003). Research desing: qualitative, quantitative. and mixed methods approaches. California, USA: SAGE Publicationsisepinternational Educational and Professional Publisher Thousand. Recuperado de: https://ucalgary.ca/paed/files/paed/2003_creswell_a-framework-for-design.pdf

Fernández, M. \& Quintero, N. (2017). Liderazgo transformacional y transaccional en emprendedores venezolanos. Revista Venezolana de Gerencia, 22(77). https://www.redalyc.org/articulo.oa?id=290/29051457005 García, M., Zerón, M. y Sáncez, Y. (2018). Factores de entorno determinantes del emprendimiento en México. Recuperado de: https://dialnet.unirioja.es/servlet/articulo?codigo $=6742098$

García, M., Zerón, M. y Sáncez, Y. (2018). Factores de entorno determinantes del emprendimiento en México. Universidad de la Rioja. 14(1). 88-103. https://dialnet.unirioja.es/servlet/articulo?codigo $=6742098$

García, Y., Mendoza, I. Romo, L. (2017). Relación Entre La Intención de la Elección de la Carrera de Ingeniería Civil y la Iniciativa Emprendedora de los Estudiantes de una Institución de Educación Superior Tecnológica en Hidalgo, México. European Scientific Journal. 13(341). 1-18. Recuperado de: https://core.ac.uk/download/pdf/328026136.pdf.

Hair, J. Ringle, C. y Sarstedt, M. (2011). PLS-SEM: indeed a silver bullet. Journal of Marketing Theory and Practice. 19(2), 139-151.

INEGI (2014). Esperanza de vida de los negocios en México. Recuperado de: http://www.inegi.org.mx/inegi/contenidos/investigacion/Experimentales/Esperanza/defaul $\underline{\text { t.aspx }}$

Kay, K. (2013). Partial least square structural equation modeling (PLS-SEM) techniques using SmartPLS. Marketing Bulletin, 24, Technical Note 1. Recuperado de: https://www.researchgate.net/publication/268449353_Partial_least_square_structural_equ ation_modeling_PLS-SEM_techniques_using_SmartPLS

ISSN: 2007-8870

Universidad de Sonora 
Kutner, M. Nachtsheim, C. y Neter, J. (2004). Applied Linear Regression Models. McGraw-Hill.

Martínez García, M. D., Salazar Hernández, B. C., \& Mendoza Moheno, J. (2020). Perfil emprendedor de los estudiantes de la licenciatura en gastronomía, en una institución de educación superior en Hidalgo. Repositorio De La Red Internacional De Investigadores En Competitividad, 13, 1653-1670. Recuperado a partir de https://riico.net/index.php/riico/article/view/1874

Meza, A. (2016). El gran problema en México de emprender sólo por dinero. Recuperado de: https://www.forbes.com.mx/gran-problema-mexico-emprender-solo-dinero/

Morante, L. (2018). Relación entre Intención Emprendedora, Norma Subjetiva, Autoeficacia Emprendedora y Personalidad Proactiva en un grupo de estudiantes de una universidad privada de Lima. Universidad Peruana de Ciencias Aplicadas (UPC), Lima, Perú. Recuperado de: https://doi.org/10.19083/ tesis/625076

Nunnally, J. y Bernstein, I. (1994). Psychometric theory. New York: McGraw-Hill.

Osorio, F. y Londoño, R. (2015). Intención Emprendedora de Estudiantes de Educación Media: extendiendo la teoría de comportamiento planificado mediante el efecto exposición. Cuadernos de Administración, 28 (51), 103-131. Recuperado de: https://www.redalyc.org/pdf/205/20543851004.pdf

Ramos, L.; López, V.; Moreno, L.; y Galván, R. (2018). La intención emprendedora en estudiantes universitarios y el proceso enseñanza aprendizaje. Revista Global de Negocios. 6(3). Pp. 1-12.

Rositas, J. (2005). Factores críticos de éxito en la gestión de calidad y su grado de presencia e impacto en la industria manufacturera mexicana. (Tesis Doctoral Inédita). Universidad Autónoma de Nuevo León. Monterrey, N.L. México.

Rositas, J. (2014). Los tamaños de las muestras en encuestas de las ciencias sociales y su repercusión en la generación del conocimiento. Innovaciones de Negocios. 11(22), 235268. Recuperado de:

http://www.web.facpya.uanl.mx/rev_in/Revistas/11_22/11.22\%20Art4\%20pp\%20235\%2 0-\%20268.pdf

ISSN: 2007-8870

Universidad de Sonora Commons Átribudión No Comercial - Sin Obra Deriva da 4.0 internacional 
Ruiz, M. Pardo, A. y San Martín, R. (2010). Modelos de ecuaciones estructurales. Papeles del Psicólogo. 31 (1), 34-45.

Tamayo, M. (2004). Proceso de la Investigación Científica. México: Editorial LIMUSA.

Recuperado de:

https://books.google.com.mx/books?id=BhymmEqkkJwC\&printsec=frontcover\&dq=meto $\underline{\text { dologia+dela+investigacion+tamayo } \& \mathrm{hl}=\mathrm{es} \& \mathrm{sa}=\mathrm{X} \& \mathrm{ved}=0 \mathrm{ahUKEwj} \text { yYrUINDQAhVLx }}$ mMKHfmdAUYQ6AEIIDAC\#v=onepage $\& \mathrm{q}=$ metodologia $\% 20 \mathrm{dela} \% 20$ investigacion $\% 2$ 0tamayo\&f=false

Toro, I. y Parra, R. (2006). Método y Conocimiento, Metodología de la Investigación.

Colombia: Fondo Editorial Universidad EAFIT. Recuperado de:

https://books.google.com.mx/books?id=4YkHGjEjy0C\&pg=PA158\&dq=investigación+no+experimental\&hl=es\&sa=X\&ved=0ahU KEwiZ8abXvNDQAhWHqlQKHbTVAwsQ6AEIGDAB\#v=onepage\&q=investigación\% 20no\%20experimental\&f=false

Torres, T. y Vidal, M. (2019). La importancia del control conductual percibido como elemento determinante de la intención emprendedora entre los estudiantes universitarios. Universidad \& Empresa. 21(37). Recuperado de: https://www.redalyc.org/jatsRepo/1872/187260206006/html/index.html

Salguero, Y. (2018). La relación de la educación empresarial con la intención emprendedora en las instituciones de educación superior del centro del país. Recuperado de: https://repositorio.uta.edu.ec/jspui/bitstream/123456789/28599/1/514\%20O.E.pdf

Sánchez García, José Carlos, Ward, Alexander, Hernández, Brizeida, \& Florez, Jenny Lizette. (2017). Educación emprendedora: Estado del arte. Propósitos y Representaciones, 5(2), 401-473. Recuperado de: https://dx.doi.org/10.20511/pyr2017.v5n2.190

Cómo citar:

Leyva Osuna, B. A., Clark Corral, J., \& Carrillo Peñuelas, D. (2021). Impacto de la Educación Emprendedora en Intención de Emprendimiento en Universidades del Noroeste de México. Revista De Investigación Académica Sin Frontera: División De Ciencias Económicas Y Sociales, (36). https://doi.org/10.46589/rdiasf.vi36.409

ISSN: 2007-8870

Universidad de Sonora 
Año 14 / Núm. 36 / julio-diciembre 2021
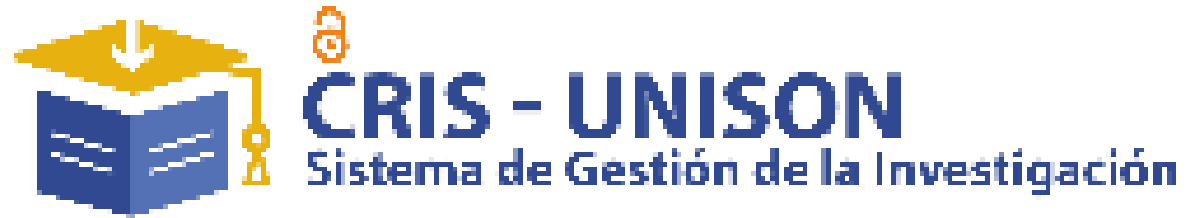

\section{Dia net}

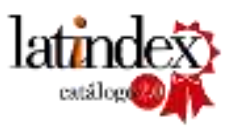

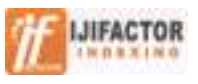

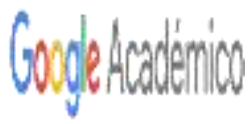

DORA

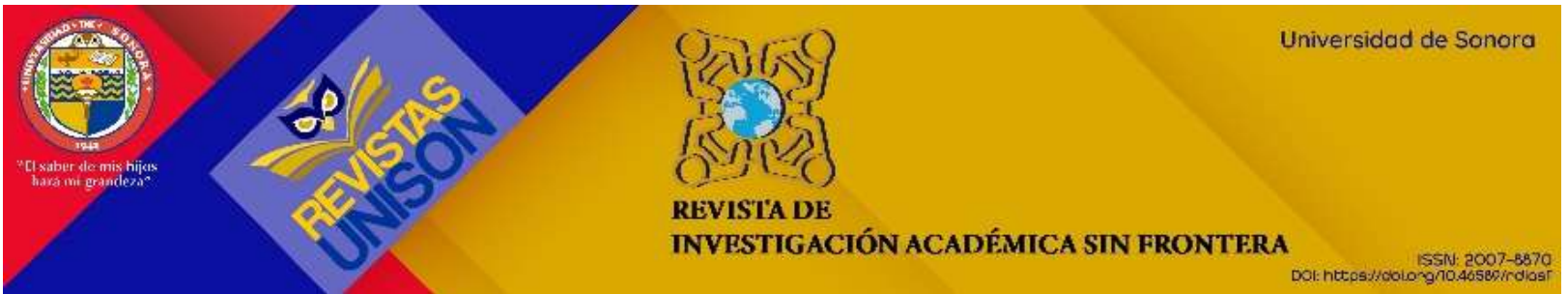

